\title{
Analysis and Experimental Study of HDFS Performance
}

\author{
Yordan Kalmukov, Milko Marinov, Tsvetelina Mladenova, Irena Valova
}

University of Ruse, 8 Studentska Str., 7017 Ruse, Bulgaria

\begin{abstract}
In the age of big data, the amount of data that people generate and use on a daily basis has far exceeded the storage and processing capabilities of a single computer system. That motivates the use of distributed big data storage and processing system such as Hadoop. It provides a reliable, horizontallyscalable, fault-tolerant and efficient service, based on the Hadoop Distributed File System (HDFS) and MapReduce.

The purpose of this research is to experimentally determine whether (and to what extent) the network communication speed, the file replication factor, the files' sizes and their number, and the location of the HDFS client influence the performance of the HDFS $\mathrm{read} / \mathrm{write}$ operations.
\end{abstract}

Keywords - HDFS, Distributed file systems, Distributed and parallel computing, Hadoop cluster.

\section{Introduction}

In the age of big data, the amount of data that people generate and use on a daily basis has far exceeded the storage and processing capabilities of a single computer system. The rapid development of the Internet technologies leads to a constant increase in the volume of structured and unstructured data, distributed worldwide across the global network.

DOI: 10.18421/TEM102-38

https://doi.org/10.18421/TEM102-38

Corresponding author: Yordan Kalmukov, University of Ruse, 8 Studentska Str., 7017 Ruse, Bulgaria Email: jkalmukov@uni-ruse.bg

Received: 24 January 2021.

Revised: 18 March 2021.

Accepted: 23 March 2021.

Published: 27 May 2021.

(cc)BY-NC-ND (C) 2021 Yordan Kalmukov et al; published by UIKTEN. This work is licensed under the Creative Commons Attribution-NonCommercial-NoDerivs 4.0 License.

The article is published with Open Access at www.temjournal.com
It is impossible for a single machine alone to store, organize, process and analyze such amount of data [4], [13].

There are two basic challenges in front of the data scientists, related to big data: how to store and process large volumes of data, and more importantly, how to extract information from these data, so that one gains a competitive advantage from them. These challenges motivate the need of building a distributed computational environment. One on the most popular and widely deployed big data storage and processing solutions is called Hadoop [5], [6]. It is a distributed environment that allows parallel processing and task execution on a cluster of commodity servers. Hadoop provides a reliable, horizontally scalable, faulttolerant and efficient big data processing service, based on the Hadoop Distributed File System (HDFS) and MapReduce.

Hadoop is an open-source project [12]. Thanks to a significant technology investment provided by Yahoo!, the Apache Hadoop has become a major corporate cloud-based technology implemented as a complete ecosystem of services. It is successfully used by many transnational corporations, such as Facebook, LinkedIn, Twitter, eBay, Samsung, J.P. Morgan, AOL and many more [4],[5]. The ecosystem includes variety of tools (Hive, Pig, HBase, Oozie, Zookeeper, Sqoop, Flume, Spark, Kafka, Impala) that allow companies to store, process and analyze large volumes of data, perform a real-time customer service, different types of optimizations, automatic email message processing, machine learning, ETL (extract, transform, load) and other operations [5], [6], [12].

The HDFS distributed file system manages files across the cluster's storage. It is responsible for data fragmentation and replication. Large files are split into multiple blocks and each of them is replicated on multiple servers, depending on the file replication factor. That guarantees data availability even if one or more servers fail. The distributed file system works in accordance with the master-slave model and implements the "Write Once, Read Many" philosophy. It is fault-tolerant, distributed "keyvalue" data storage, optimized to handle large data files [1], [2], [12]. In contrast to the regular file 
systems, the HDFS does not support file editing. Existing files could just be appended with new data, but not be edited. The HDFS is designed to reliably store large files of constant (immutable) data which are written once and read many times [7], [8].

Since HDFS plays an important role in the entire Hadoop ecosystem, its performance (represented by the throughput of read/write operations) should be studied in details [3], [16], [17]. More precisely it is necessary to determine the dependence of the HDFS $\mathrm{read} /$ write performance on the size of the input data [7], [15]. Such an analysis will help to evaluate the efficiency of HDFS and to identify the major factors that influence on its performance.

The purpose of this research is to experimentally determine whether (and to what extent) the following factors influence the performance of HDFS input/output operations:

- Network communication speed between cluster's nodes;

- File replication factor;

- File size and number of files;

- Location of the HDFS client inside the cluster.

Before performing any experiments, it is worth to do some preliminary theoretical analyses. They will help in experiment planning and serve as starting hypotheses that experiments should prove or reject.

The article is structured as follows: Section 2 reviews some related research. Section 3 provides a detailed analysis of the HDFS read and write operations. Section 4 presents a comprehensive experimental study of the HDFS performance and the major factors that affects it. Finally, in Section 5, the authors summarize their observations and conclusions as a result of the experiments, and give some ideas for further research.

\section{Related Work}

With the rapid development of the big data processing systems during the last years, various tools have been created for their evaluation and benchmarking, in order to identify bottlenecks and provide further improvement.

Han etal. [13] review the most modern methods of comparing big data storage and processing systems and use popular open-source benchmarks to evaluate their performance. They provide a good overview of the three important aspects of benchmarking - load generation techniques, input data generation techniques and systems' evaluation indicators.

Krishna et al. discuss in [1] the efficiency of read and write operations in HDFS in terms of file sizes small and large data files. They use a five-node Hadoop cluster to evaluate the HDFS performance.
Their results show that HDFS performs well for files larger than the default block size, but not that good and efficient for files smaller than the block size. However their conclusions are made based on a small number of cluster nodes and without taking the replication factor into account. A similar study could be found in [2], where authors compare the performance of the distributed file system (HDFS) in respect to the local file system (LFS). The HDFS provides an efficient, fault-tolerant storage and processing environment for large files, while the LFS could handle small files efficiently as well (but with no fault tolerance).

Articles [3], [11] present various models for HDFS performance evaluation. Due to the complexity of data organization and the DFS working principles, modeling HDFS performance is hard to do, especially if we take into account the many factors that could influence it. Dong et al. [3] study the dependence of the HDFS read/write execution time on the size of the input file, in order to obtain HDFS performance models from a systematic point of view. Authors define metrics of dynamic characteristics of the HDFS operations. Their approach helps not only to predict the HDFS performance on certain conditions, but also to identify the major factors that affect it, as well as the relationships between these factors.

The Hadoop distributed file system is very efficient in processing large files, but in case of a large number of small files its performance rapidly drops since all files are managed by a single server. While storing a file, a map task is created. Due to the large number of small files, a large number of map tasks should be created that reduces the file system performance. Issues related to storing small files in HDFS are reviewed in multiple studies [8], [9], [10]. Various methods of solving them have been recently proposed in the literature. Rathidevi and Srinivasan [7] present a comparative analysis of these methods. Bok et al. [8] propose a distributed caching schema, providing efficient access to small files in HDFS. It reduces the amount of metadata managed by the name node by combining multiple small files in a single storage block.

Shafer et al. [14] study bottlenecks in distributed file systems' performance from an architectural point of view. The aim is to evaluate the trade-offs between portability and performance in HDFS. Vaidya and Deshpande [15] perform a comparative analysis of the open-source distributed file systems "GlusterFS", "Luster", "Ceph" and "HDFS" based on criteria such as fault-tolerance, influence of the replication factor, security and optimization of small files management. Their research was done using MapReduce technologies. 


\section{Overview of HDFS read and write operations}

The two basic operations related to HDFS are uploading (write) and downloading (read) files to/from the distributed filesystem. There are generally two ways to access the HDFS filesystem:

- By using the Command Line Interface (CLI) of the "built-in" client, which is provided together with the Hadoop distribution. It is called "FileSystem Shell" and is implemented in the hdfs program, situated in the Hadoop/bin directory;

- By the WebHDFS REST API.

The web API allows external applications to interact with the HDFS and read/write files to it. However for benchmarking purposes the command line interface, provided by the built-in client is better. It can utilize most of the hardware resources and for that reason is perfectly suitable for finding bottlenecks in the entire system. The client could be run from any cluster node - name node or data node. Since all nodes use the same Hadoop distribution, the client is located in the same directory and is used in the same manner everywhere.

Mentioning bottlenecks that could influence HDFS performance, the two most obvious are: network communication speed between servers and data transfer speed of the servers' hard drives. These two are both equally important. If the network's speed is low then replicas will be stored and read slowly, but the same is true if hard drives are slow as well. Not only the type and speed of the hard drives matter, but also their configuration. If drives are configured in RAID 0 or RAID 10, that could almost double their data transfer speeds. A network speed of $1 \mathrm{Gbit} / \mathrm{s}$ is commensurate with rotating mechanical hard drives configured in RAID 10. The drives will be just a bit faster. However if the network speed is increased to $10 \mathrm{Gbit} / \mathrm{s}$, rotating hard drives will be the bottleneck for sure, since their transfer speed is 7-8 times lower than the network's capabilities. In case of high network speeds, such as $10 \mathrm{Gbit} / \mathrm{s}$ and higher, it is mandatory that data nodes use SSD drives. Not SATA SSD drives, but NVMe SSD drives, since SATA standard is also limited to $6 \mathrm{Gbit} / \mathrm{s}$, while PCIe 4.0 NVMe supports up to $64 \mathrm{Gbit} / \mathrm{s}$.

The process of uploading (writing) a file in the HDFS is described on Figure 1. Initially, the client interacts with the name node by sending a create/write request (step 1). The name node checks if the user has privileges to upload files in the specific HDFS location and if there is a file already with the same name. In case user privileges are sufficient and the file is not duplicated, the name node returns a list of all data nodes' addresses where the user can store his/her data. Then the client splits the file into blocks of $128 \mathrm{MB}$ each (by default, but may vary). To achieve a fault tolerance and high data availability, each block is replicated several times (3 by default, but could be configured) on different data nodes. The client communicates directly with the first data node only (step 2). Then replicas are automatically copied from data node to data node (steps 3.x). It is important to note here that blocks are being sent directly from the client to the data nodes. They do not pass through the name node at all. The latter is just a data/block catalogue. It manages the overall communication and determines where individual blocks should reside, but does not route them. After a data node receives a replica from another data node (or the client), it sends an acknowledgement back to the sender verifying the replica is saved successfully (steps 4.x). Once the client got an acknowledgment for the last block of the file, it connects to the name node again sending a complete signal (step 5), notifying the name node that the process of uploading (writing) is completed successfully. According to Data Flair, an IT training company, some amount of blocks (depending on the number of hardware threads) could be processed in parallel, boosting the overall HDFS performance. Our observations and experimental analyses support this statement as well.

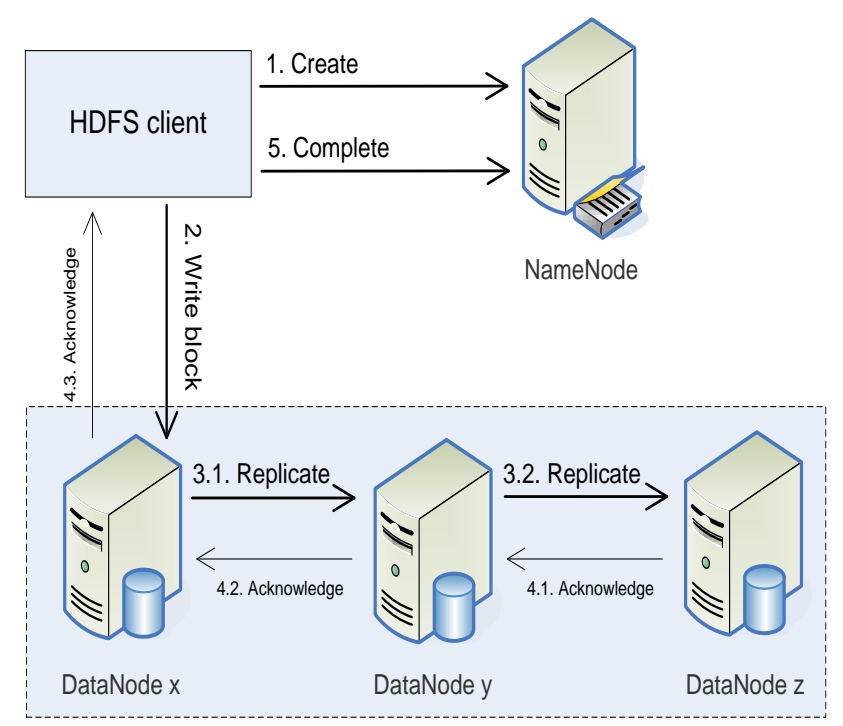

Figure 1. HDFS data write workflow

The process of reading a file from the HDFS filesystem is illustrated on Figure 2. Initially, the client connects to the name node and requests a list of all data nodes that store replicas, building the requested file (step 1). The name node first checks if the user has privileges to download the file. If yes, it returns a list of all blocks and the location of their replicas. Then the client starts reading blocks from the data nodes directly (steps 2 and 3). Data blocks do not pass through the name node. The client reads each block from just a single replica (data node) - 
the one that is closest to the client. It is worth mentioning that "closest" is a relative notation. It reflects communication speed and time rather than physical distance. Once the client has read all requested blocks, it merges them to reconstruct the downloaded file.

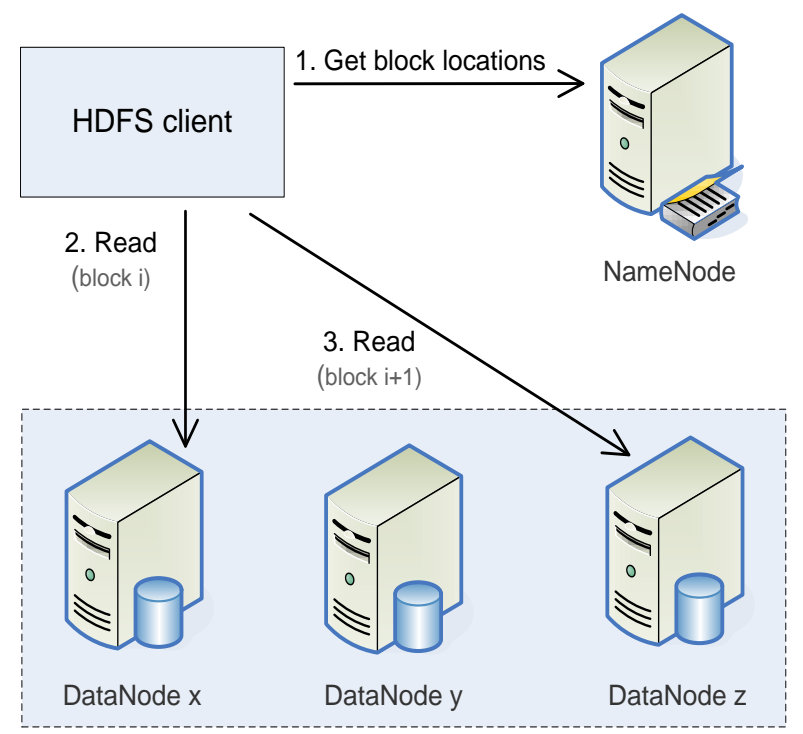

Figure 2. HDFS file read workflow

The theoretical analysis leads to some important assumptions that section 4 is aiming to experimentally prove or reject:

- The network communication speed is crucial for HDFS performance; however hard drives' transfer speed is equally important as well.

- Since the client communicates directly with the first data node only, when writing a file, the replication factor (the number of replicas per block) should not influence the uploading time. However, higher replication factor will increase the cumulative network traffic. If the network hub/switch/router cannot handle that, it may lower the speed.

- As the client reads each block just from a single replica, then increasing the replication factor (the number of replicas per block) should not influence the downloading speed at all. Higher number of replicas will increase the faulttolerance, but not influence the download time.

\section{Experimental Evaluation of HDFS Performance}

This section aims to experimentally prove or reject the previously formulated assumptions.

To increase objectivity of the analysis and reduce the influence of the so-called random or uncontrollable factors (variables) each test is replicated/repeated 3 times, then results are averaged. Among the most significant random factors is the cluster's current load, determined by the number of other users and tasks running at the same time. That also includes some "Cloudera Manager"-related tasks which are being initiated and executed automatically, meaning they could not be predicted. However, repetitions could not happen sequentially one after another, since HDFS caches the read data and every measurement after the first one will be false. For that reason, we have done each repetition on a different day. Meanwhile other colleagues have used the cluster for other tasks. Tests in all experiments have been repeated 3 times, except for the experiment at $100 \mathrm{Mbit} / \mathrm{s}$ network's speed that has been performed just once, as it took too much time to complete.

Experiments are made on the following hardware:

NameNode - 1 unit (HPE ProLiant DL360 Gen10):

CPU: 2 x Intel Xeon Silver $4110 @ 2.1 \mathrm{GHz}, 8$ cores, 16 threads; (total threads: 32 )

RAM: 64 GB DDR4 ECC @2666 MHz

HDD: 2 x 1 TB, HPE Serial Attached SCSI @ 15000 rpm, configured on RAID 1

Network: Broadcom NetXtreme BCM5719 Gigabit Ethernet, $1 \mathrm{Gbit} / \mathrm{s}$

Hadoop version: 3.0.0 (CDH 6.3.2) on CentOS 7.8

DataNodes - 9 units (HPE ProLiant DL20 Gen10):

CPU:Intel Xeon E-2124@3.30 GHz,4 cores,4 threads RAM: 16 GB DDR4 ECC @2666 MHz

HDD: 4 x 2 TB, HPE Serial Attached SCSI @15000 rpm, configured on RAID 10

Network: Broadcom NetXtreme BCM5720 Gigabit Ethernet, $1 \mathrm{Gbit} / \mathrm{s}$

Hadoop version: 3.0.0 (CDH 6.3.2) on CentOS 7.8

Network switch: HPE OfficeConnect 182024 Port Gigabit switch, $1 \mathrm{Gbit} / \mathrm{s}$ per port.

For test data we use dummy files generated by the operating system (with the dd utility). We actually do not care about the files' content, but we need to precisely manage their sizes in order to perform an accurate and objective experimental analysis.

\section{A. Initial experiment - determine read/write time at 1 Gbit/s network speed, replication factor 3 and client run on the name node}

The initial experiment is to determine upload and download time of single large files, ranging from 1 to 512 gigabytes (GB). Each test is doubling the file size of the previous one, as shown on Figure 3. Numbers given under the $\mathrm{x}$-axis represent the averaged times from all three repetitions of the respective test. Y-axis represents download/upload (read/write) time in seconds on a logarithmic (base 2) scale. Logarithmic y-axis is needed since data points on the $\mathrm{X}$-axis are equally distanced, but represent doubling the size (more precisely 2 to the power of).

Results are obtained with $1 \mathrm{Gbit} / \mathrm{s}$ network speed between nodes, default replication factor of 3 and 
client run on the name node. They clearly show a linear dependency of the execution time on the size of the input data, even for a large amount of data. The linear complexity proves that the HDFS is indeed an efficient distributed file system.

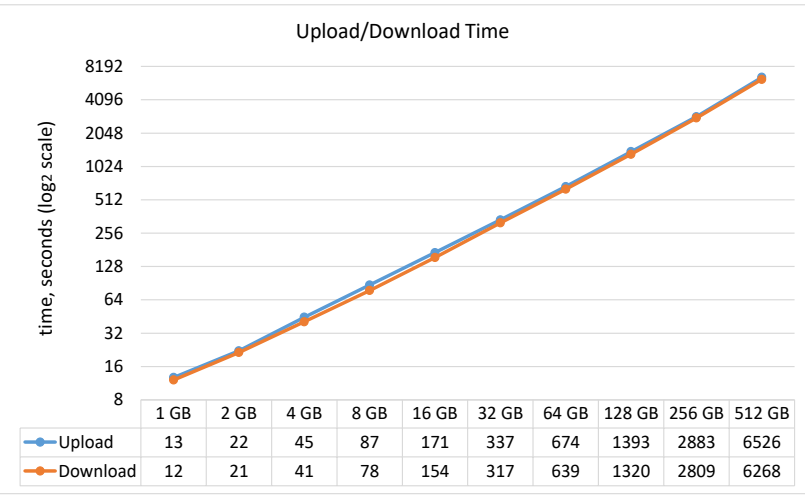

Figure 3. Dependence of the HDFS read/write time on the size of input data

As observed, upload and download times are quite similar (upload is a bit slower) suggesting that block replication on multiple nodes does not put much higher load. Of course, we should note here that our network switch supports 1 Gbit/s per port, independently on the other ports, so replicas could be distributed in parallel without lowering the node-tonode communication speed.

The work of the Hadoop cluster, in general, or the HDFS, in particular, could be also revealed by the "Cluster Disk IO", "HDFS IO" and "Cluster Network IO” diagrams, provided by Cloudera Manager.

The cluster disk IO (figure 4) shows cumulative disk read (blue) and write (green) transfer rates for the entire cluster. The first plateau (4:37 to 4:59) corresponds to reading files from the HDFS. Since each block is read from a single replica, the total bytes read from the data nodes (blue) are equal to the total bytes written to the client's disk (green). The second plateau (5:00 to 5:24) is related to writing files in HDFS. You can easily notice that bytes written to the cluster's disks (green) are three times more than ones read from the client (blue). That is because each block is written (replicated) to three different data nodes. The same could be observed from the Cluster Network IO diagram (figure 5) as well. Network speed when downloading (first plateau) is close to $1 \mathrm{Gbit} / \mathrm{s}$, while during upload, the cumulative network speed gets 3 times higher as data are written to 3 servers in parallel.

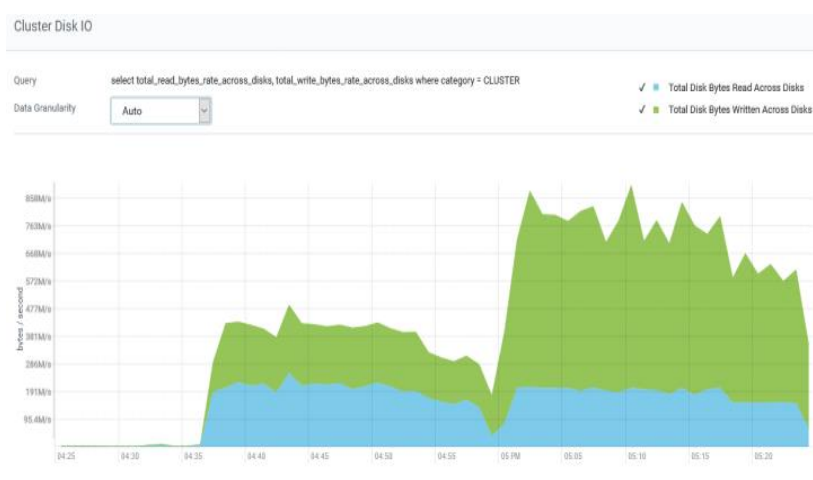

Figure 4. Cumulative cluster disk transfer rates during file download (first plateau) and file upload (second plateau)

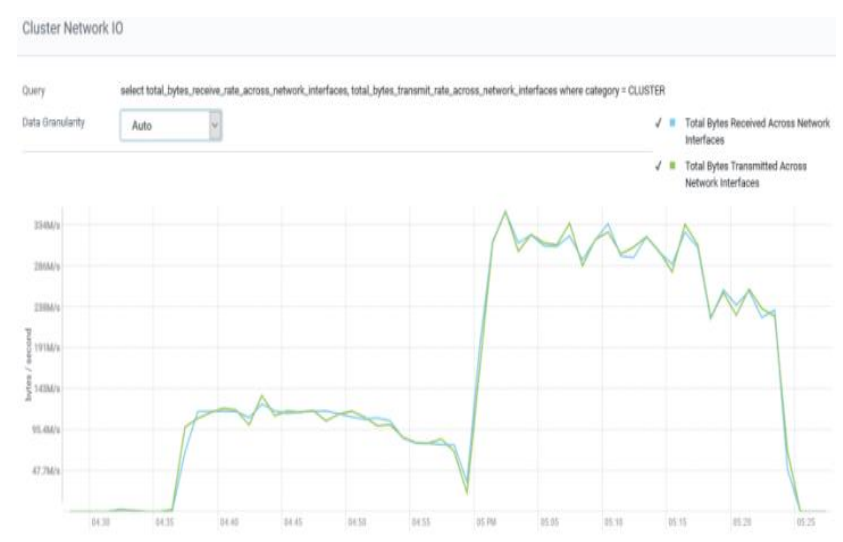

Figure 5. Cumulative cluster network speed during file download (first plateau) and file upload (second plateau)

\section{B. Influence of the client's location (name node or data node)}

We performed all experiments by using the command line interface of the built-in client (FileSystem Shell) which comes together with the Hadoop distribution. However, it can be run from any cluster node, regardless it is a name node or a data node. This experiment aims to check if there is any difference. Of course hardware performance matters, but beside that it is interesting if location does as well. So we have replicated Experiment A, with the same data and the same settings, but run from different data nodes in order to compare results with those of A. Results are quite interesting. When downloading/reading files, there is no difference if the client runs from name node or data nodes - times are almost identical. However uploading from the data node is about $40 \%$ slower than uploading from the name node. That could be explained by different hardware performance of the client's machine - the name node has 32 cores and 64 GB RAM, while data nodes has just 4 cores and 16 GB RAM. As described in section 3 , the file is being split into multiple blocks on the client machine and these blocks could be processed in parallel, depending on the number of hardware threads. So, client's hardware performance really matters. 
Besides the expected influence of the hardware performance of the client's machine, we notice something quite intriguing. When uploading a file to the HDFS, if the client runs on a data node, one of the replicas will be stored for sure on the same data node. That could be observed and proven in two ways - by using the "HDFS IO" and the "Cluster Network IO" diagrams (figure 6); and by checking replicas' locations with the Hadoop's "DFS Health" tool.

Figure 6 shows a collage of both HDFS IO (upper part) and Cluster Network IO (lower part) diagrams during the same download/upload session. The first plateau (the blue one) corresponds to the total bytes read from data nodes during download. The second plateau (the green one) represents total bytes written across data nodes during upload of the same files. As already mentioned, upload from our data nodes is about $40 \%$ slower due to the lower number of hardware threads. That's why the width of the green shape is greater than the width of the blue one. But that is not important. Notice that the write transfer rate (the green plateau) during upload is just 2 times higher than the read rate during download. It proves that just 2 replicas are transmitted to other data nodes while the 3-rd one stays within the same data node. As a second proof we use the Hadoop's DFS Health tool to check the locations of blocks' replicas. It shows that a replica of every block is stored within the same data node, where the client has been run on.

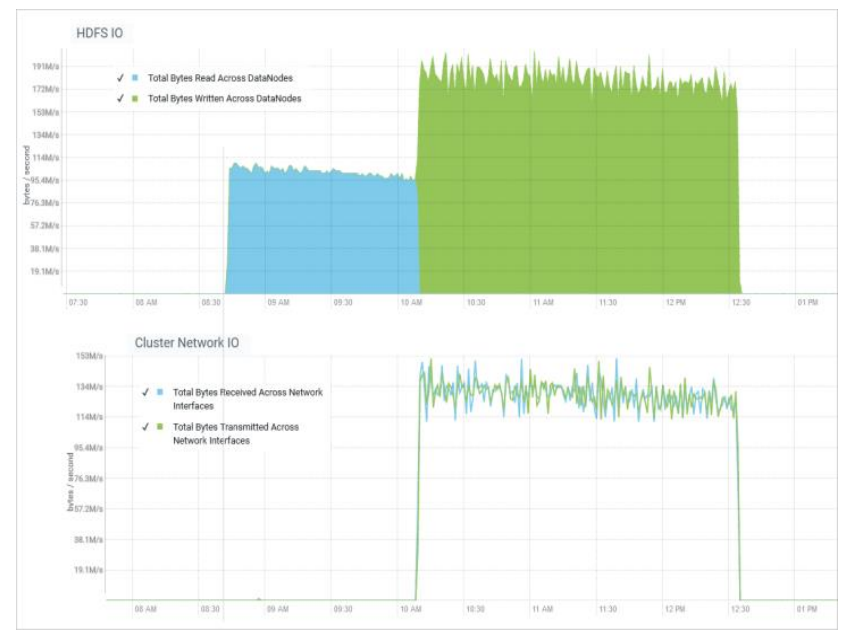

Figure 6. A collage of HDFS IO (upper part) and Cluster Network IO (lower part) diagrams during the same read/write session, showing that 2 (out of 3) replicas are being transited during upload from a data node, and there is no network activity during download at all.

The fact that when uploading a file from a data node, one of the replicas will be stored on it has an important consequence. As described in section 3, when downloading a file each block is read just from a single replica - the closest one. The closest replica is the one situated on the same machine. So, if a file has been uploaded by a client that runs on data node $X$, then $X$ will have replicas of all blocks building the file. Subsequently, if the file is being downloaded/read by a client that runs on the same data node $X$, then the client will use local replicas (from X) only. In other words - if a file is being downloaded from a data node, from which it has been previously uploaded, the file will be reconstructed from local replicas only, without causing any network activity. In this case, network communication speed does not matter at all. This is also proven on figure 6 . Have a look at the download session from 8:40 to 10:10 a.m. (the blue shape). About $100 \mathrm{MB} / \mathrm{s}$ are constantly read from the HDFS during that time, but there is no network activity at all (the lower part of the figure - Cluster Network IO), during this period. Why? This is because during download; the client has been using local replicas only.

\section{Influence of the network communication speed}

Since the HDFS is a distributed filesystem that stores multiple replicas on multiple physical servers, it is obvious that network communication speed is a performance key point. Our network equipment supports maximum speed of $1 \mathrm{Gbit} / \mathrm{s}$, so we have made two experiments that share the same input data, replication factor and other settings, but run on different network speeds $-1 \mathrm{Gbit} / \mathrm{s}$ and $100 \mathrm{Mbit} / \mathrm{s}$. It is worth to mention that $100 \mathrm{Mbit} / \mathrm{s}$ is an obsolete and unrealistic speed for LAN network, but pretty representative for a WAN network, spread over large territory or the Internet. Results show that read time on $1 \mathrm{Gbit} / \mathrm{s}$ network (blue line on figure 7 ) is about 7 times lower (on average) in comparison to 100 Mbit/s network (the orange line). We actually expected the difference to be a bit higher since there is a 10-times change in communication speed, but results suggest that probably rotating hard drives are the actual bottleneck in the $1 \mathrm{Gbit} / \mathrm{s}$ system. The dependency of execution time on the size of input data however stays linear.

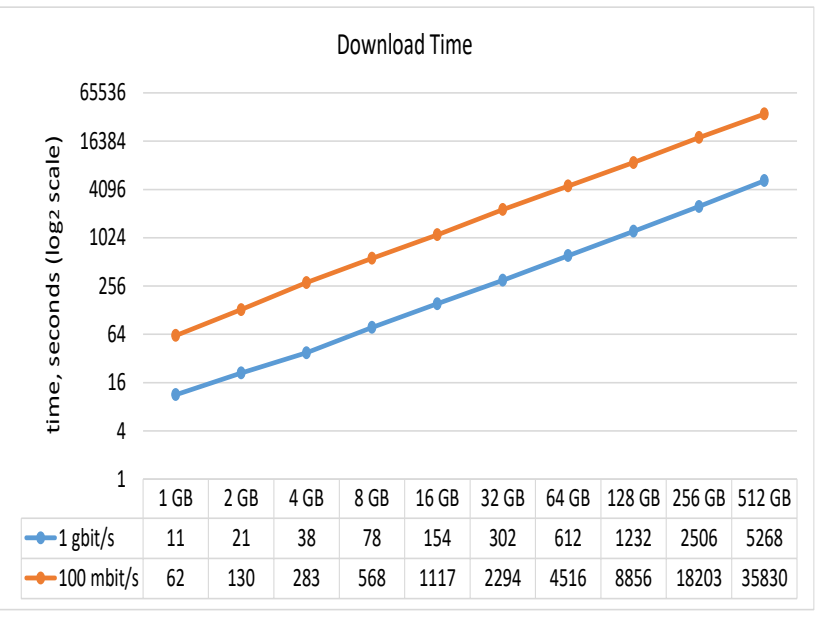

Figure 7. HDFS read times on

$1 \mathrm{Gbit} / \mathrm{s}$ network vs. $100 \mathrm{Mbit} / \mathrm{s}$ network

Results are quite similar for writing files as well.

\section{Influence of the block replication factor}

According to the preliminary theoretical analysis in section 3, data replication should not influence the downloading/read time at all, since each block is read 
just from a single replica. Experiments prove this assumption is fully correct (Figure 8). Blue line represents read time at replication factor 6 (i.e. each block is replicated on 6 different data nodes), while the orange one is at replication factor 3. As seen they almost perfectly match. So, indeed the number of replicas does not influence the file read speed. All experiments are performed on $1 \mathrm{Gbit} / \mathrm{s}$ network and client that runs on the name node.

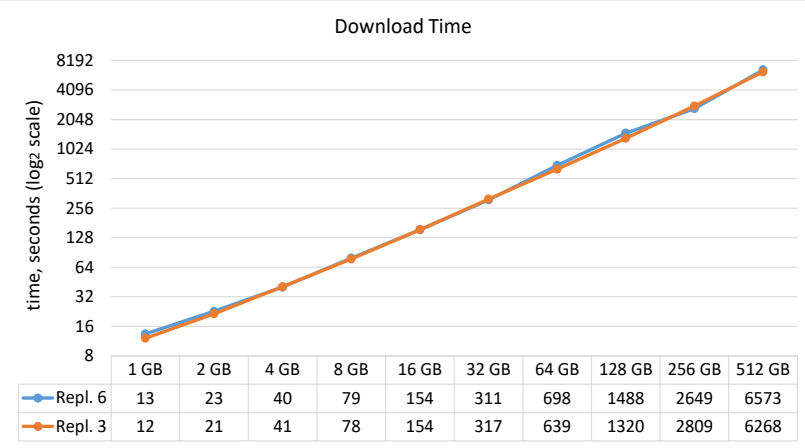

Figure 8. Dependence of the HDFS read time at replication factors 3 and 6 on the size of input data. As lines almost match, replication factor does not influence the HDFS read performance.

When writing files however the situation is different as the higher number of replicas that should be distributed in parallel, significantly increases the cluster's cumulative network traffic. In this case the network switching devices become crucial. If they support their listed speed per port, independent on the other ports, they may handle the traffic and thus the higher level of replication may not influence the uploading speed. However if the computing power of these devices is not enough to handle the increased traffic, they may lower their overall network speed, thus decreasing the cluster's performance. Results of our experiments show our network switch performs well, so that writing time at replication factor 6 (the blue line on figure 9) almost matches the one at replication factor 3 (the orange line).

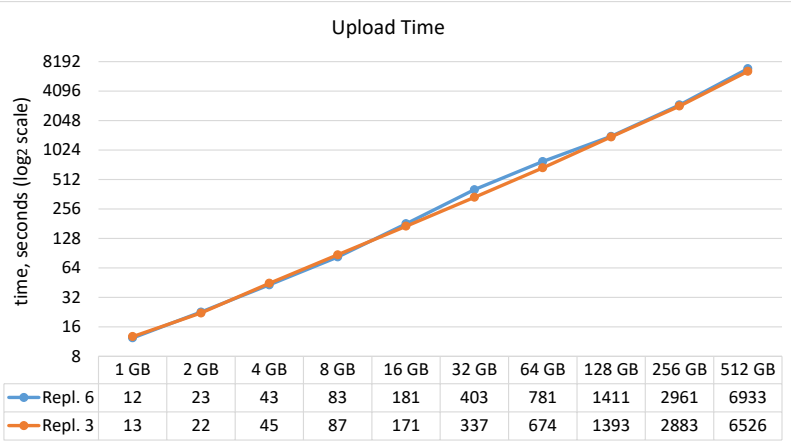

Figure 9. Dependence of the HDFS write time at replication factors 3 and 6 on the size of input data. As lines almost match, replication factor does not influence the HDFS write performance.
The cluster Disk IO and Cluster Network IO diagrams generated by Cloudera Manager visually show the increased cumulative data transfer of both network and hard drives (Figure 10). The total bytes written (green) are 6 times more than the total bytes read (blue) since each block is sent to 6 different data nodes. The cumulative network traffic goes over 600 $\mathrm{MB} / \mathrm{s}$ with a peak of $652 \mathrm{MB} / \mathrm{s}(5.2 \mathrm{Gbit} / \mathrm{s})$ at 11:11:29. It is a result of loading 6 network ports of the switch in parallel, although all of them support up to $1 \mathrm{Gbit} / \mathrm{s}$ individually.
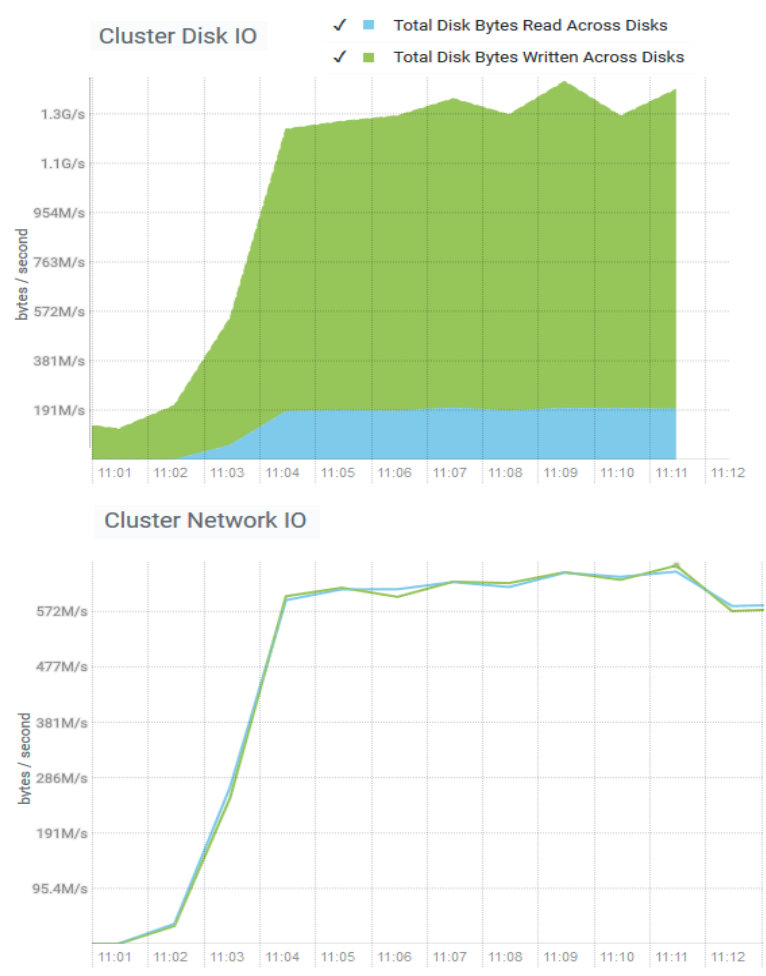

Figure 10. A collage of Cluster Disk IO (upper part) and Cluster Network IO (lower part) diagrams, showing that 6 replicas are transmitted in parallel during HDFS write operations at replication factor 6.

\section{E. Influence of the number of files and their sizes}

As we know from everyday usage of network communication and different types of file transfer, copying single large files is much more efficient than copying multiple smaller files. There are objective reasons for that, including metadata overheads. As described in section 3, large files in HDFS are split into multiple blocks of $128 \mathrm{MB}$ and each block is transferred and replicated separately. So, we cannot have a single instance larger than $128 \mathrm{MB}$. But what if files are smaller than that? Will they occupy the entire block of $128 \mathrm{MB}$ ? Will they be copied slower? To answer these questions we performed a series of three experiments. All of them at replication factor 3 on $1 \mathrm{Gbit} / \mathrm{s}$ network. The first experiment reading/writing single large files are of $16 \mathrm{~GB}, 32$ GB, 64 GB, 128 GB and 256 GB. Second experiment - copying absolutely the same amount of data per 
test, but on multiple files about 102.4 MB each. Third experiment - copying the same amount of data, but on multiple files of about $10.24 \mathrm{MB}$ each, i.e. 10 times more files in comparison to experiment 2, while keeping the total data size per test the same.

How have we chosen files' sizes? For the second experiment we wanted to have 10 files per GB, so that for $256 \mathrm{~GB}$ we have 2560 files. That is around 102.4 MB per file. It should be mentioned that the total sum of bytes of all files exactly matches (to byte) the size of the corresponding single large file. Furthermore $102 \mathrm{MB}$ is close to the block size of 128 $\mathrm{MB}$, so it could be expected the performance difference will be not much. For the third experiment we wanted to have 100 files per GB, i.e. 25600 files for $256 \mathrm{~GB}$. That is around 10.24 MB per file. This value is much lower than the block size and thus provides good opportunities for performance analyses related to computational time and storage space.

Results are illustrated on Figure 11 for writing files to HDFS and figure 12 for reading files. As expected, the difference in time when comparing single large files to multiple $102 \mathrm{MB}$ files is not that much, especially for smaller data sizes. Large files are automatically split to multiple files of size $128 \mathrm{MB}$. For small data sizes (for example up to $16 \mathrm{~GB}$ ), the number of blocks of a single large file is similar to the number of multiple $102 \mathrm{MB}$ files that results in almost similar upload/download time. However as data size increases, the difference between the number of blocks of the large file and the number of $102 \mathrm{MB}$ files increases as well. For example, a single 16 GB file is split into 128 blocks, while the number of $102 \mathrm{MB}$ files for the same total size is 160 , or a difference of 32 . A single $256 \mathrm{~GB}$ file is split into 2048 blocks, while the number of $102 \mathrm{MB}$ files for the same $256 \mathrm{~GB}$ is 2560 , or a difference of $\underline{512}$ ! That is why with increasing the input data size, the two lines (the blue and the orange) diverge and the time difference gets rapidly higher, as many more blocks should be processed with smaller files.

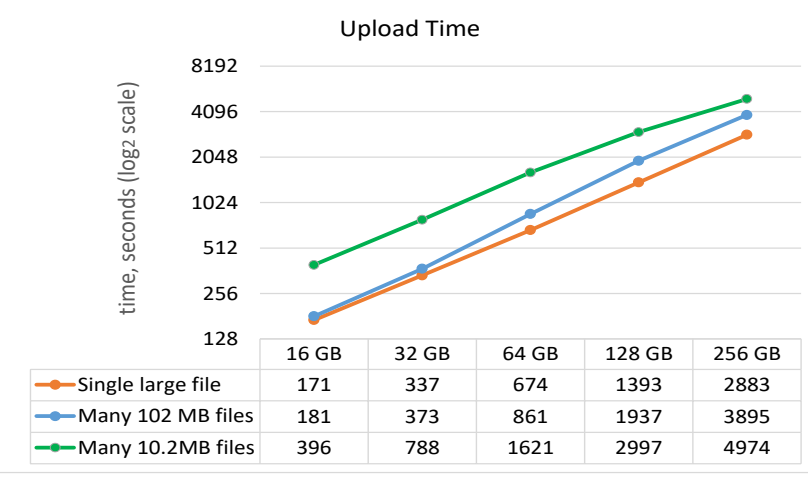

Figure 11. Dependence of the HDFS write performance on the size and the number of files

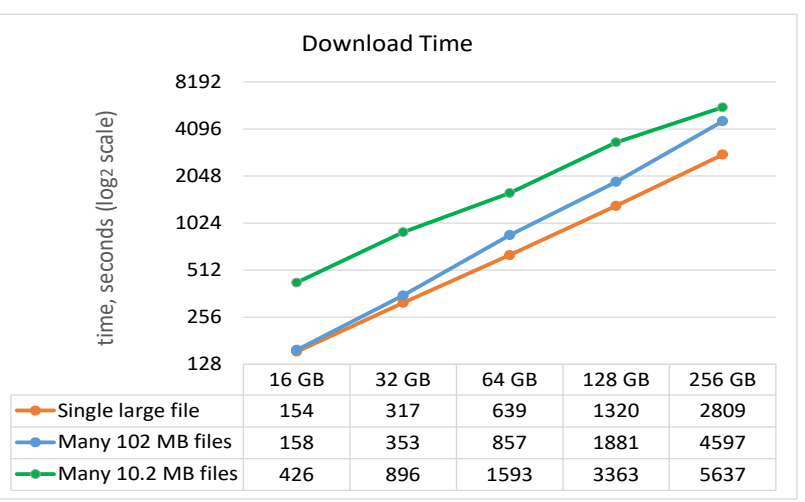

Figure 12. Dependence of the HDFS read performance on the size and the number of files

The most interesting case however is the green line - many more even smaller (10.24 MB) files. Here, on small data sizes (16 to $64 \mathrm{~GB}$ ), the read/write time of multiple 10.24 MB files is much (times) higher since there are 12 files (blocks) per each block of the large file. However with increasing the input data size, the green line starts to converge with the blue one, suggesting that the number of blocks for these two cases has become large enough not to make a significant difference.

Another interesting aspect of working with small files is whether a file occupies the entire 128MB block when stored in the filesystem or $128 \mathrm{MB}$ is actually the maximum size, but the block is not fixed to that value. Our observations, by using the hdfs -du command during experiments, clearly show that $128 \mathrm{MB}$ is the maximum block size, but if a file is smaller, it consumes as much HDFS space as its size multiplied by the replication factor.

\section{Conclusions}

After performing dozens of experiments and subsequent analysis of the results, we can conclude that:

- Network communication speed is crucial for HDFS performance, but it is not the only bottleneck. Hard drives' data transfer rates are also very important, especially in high-speed local or metropolitan area networks.

- When uploading/writing a file to the HDFS, if the client runs on a data node, one of the replicas will be surely stored on the same data node.

- When reading a file from the HDFS, if the client runs on a data node, and there is a block replica stored on the same data node, then the client will use its local replica.

- As a result of the previous two - if a file is being downloaded from a client on a data node, from which it has been previously uploaded, then the file will be reconstructed from local replicas only, without causing any network activity. In this case, network communication speed does not matter at all. 
- When reading a file from the HDFS, the replication factor does not influence the reading speed/time at all as each block is read from a single data node only.

- When writing a file to the HDFS, the replication factor does not influence the performance directly, however higher replication results in multiplied simultaneous network traffic. If the network communication devices cannot handle the increased traffic that may decrease the file upload speed.

- Reading/writing many small files is much slower than reading/writing the same amount of data in a single large file or a few large enough files. The reason is that many more blocks should be processed with smaller files.

- The block size (128MB by default) is actually a maximal block size, but if a file is smaller than that, it occupies as much HDFS space as its size is.

- Since large files are split to multiple blocks of $128 \mathrm{MB}$ each which could be processed in parallel, the number of hardware threads on the client is important to reach optimal performance.

As HDFS is a basis of all other services of the Hadoop Ecosystem, we plan to extent our research and experimental evaluation to other services, such as Hbase and Hive, as well. They both could be used as reliable and high-performance data storage.

\section{References}

[1]. Krishna, T. L. S. R., Ragunathan, T., \& Battula, S. K. (2014, July). Performance evaluation of read and write operations in hadoop distributed file system. In 2014 Sixth International Symposium on Parallel Architectures, Algorithms and Programming (pp. 110-113). IEEE.

[2]. Srinithya, L., \& Reddy, G. V. R. (2014). Performance Evaluation of Hadoop Distributed File System and Local File System. International Journal of Science and Research, 3(9), 1174-1183.

[3]. Dong, B., Zheng, Q., Tian, F., Chao, K. M., Godwin, N., Ma, T., \& Xu, H. (2014). Performance models and dynamic characteristics analysis for HDFS write and read operations: A systematic view. Journal of Systems and Software, 93, 132-151.
[4]. Marz, N., Warren, J. (2015). Big Data: principles and best practices of scalable real-time systems, Manning Publications, Shelter Island, NY, USA.

[5]. Grover, M., Malaska, T., Seidman, J., \& Shapira, G. (2015). Hadoop application architectures: Designing real-world big data applications. " O'Reilly Media, Inc.".

[6]. McCreary, D., \& Kelly, A. (2014). Making sense of NoSQL. Shelter Island: Manning, 19-20.

[7]. Rathidevi, R., Srinivasan, S. (2018). Small files problem in Hadoop - a survey. International Journal of Pure and Applied Mathematics, 119(15), 28332841.

[8]. Bok, K., Oh, H., Lim, J., Pae, Y., Choi, H., Lee, B., \& Yoo, J. (2017). An efficient distributed caching for accessing small files in HDFS. Cluster Computing, 20(4), 3579-3592.

[9]. Rathidevi, R., Parameswari, R. (2018). A systematic approach for merging small Files in Hadoop using prolonged HDFS framework. International Journal of Advanced Studies of Scientific Research, 3(12), 408-413.

[10]. Bende, S., \& Shedge, R. (2016). Dealing with small files problem in hadoop distributed file system. Procedia Computer Science, 79, 1001-1012.

[11]. Cho, J. Y., Jin, H. W., Lee, M., \& Schwan, K. (2014). Dynamic core affinity for high-performance file upload on Hadoop Distributed File System. Parallel Computing, 40(10), 722-737.

[12]. Lublinsky, B., Smith, K. T., \& Yakubovich, A. (2013). Professional hadoop solutions. John Wiley \& Sons.

[13]. Han, R., John, L. K., \& Zhan, J. (2018). Benchmarking Big Data Systems: A Review. IEEE Computer Architecture Letters, 11(03), 580-597.

[14]. Shafer, J., Rixner, S., \& Cox, A. L. (2010, March). The hadoop distributed filesystem: Balancing portability and performance. In 2010 IEEE International Symposium on Performance Analysis of Systems \& Software (ISPASS) (pp. 122-133). IEEE.

[15]. Vaidya, M., \& Deshpande, S. (2016). Critical study of performance parameters on distributed file systems using MapReduce. Procedia Computer Science, 78, 224-232.

[16]. Zhao, D. (2017). Performance comparison between Hadoop and HAMR under laboratory environment. Procedia computer science, 111, 223229.

[17]. Bakratsas, M., Basaras, P., Katsaros, D., \& Tassiulas, L. (2018). Hadoop mapreduce performance on ssds for analyzing social networks. Big data research, 11, $1-10$. 